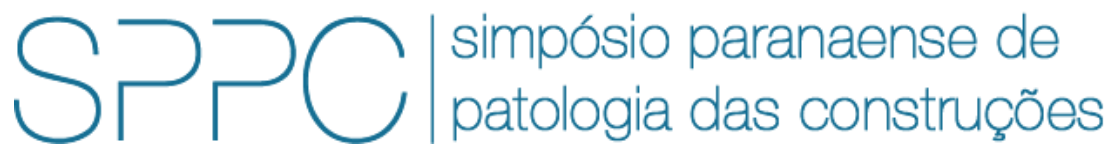

ISSN 2526-7248 artigo 2SPPC1017, pp. 199-212, 2017

\title{
Análise do índice de chuva dirigida em cidades do Paraná e sua importância no projeto de fachadas de edifícios
}

\author{
Gustavo Dias Medeiros Batista ${ }^{1}$, Leonardo Torterolli Rufato², Dayane de Cristo Miranda ${ }^{3}$, Dominique \\ Elena Giordano 4 e Marcelo Henrique Farias de Medeiros ${ }^{5}$ \\ 1 Graduando, Universidade Federal do Paraná,gustavo_d_medeiros@hotmail.com \\ 2 Graduando, Universidade Federal do Paraná, leorufato@gmail.com \\ ${ }^{3}$ Graduanda, Universidade Federal do Paraná, daymiranda05@gmail.com \\ ${ }^{4}$ Mestre, Instituto Federal do Paraná, domi.giordano@gmail.com \\ ${ }^{5}$ Doutor, Universidade Federal do Paraná, medeiros.ufpr@gmail.com
}

\begin{abstract}
Resumo: Tendo em vista a progressiva preocupação com a durabilidade das construções, torna-se necessário estabelecer métodos para análise da influência das condições ambientais sobre as estruturas. Neste contexto, este trabalho visa o estudo do fenômeno de chuva dirigida (chuva associada ao vento) e sua influência sobre a durabilidade de fachadas. A análise baseou-se nos dados de três estações metereológicas do estado do Paraná, localizadas nas cidades de Londrina, Maringá e Curitiba, buscando identificar as orientações de chuva dirigida mais criticas destas cidades. Analisouse a variação da chuva dirigida ao longo do tempo através dos Índices de Chuva Dirigida Anual, Mensal e Direcional, que indicam, respectivamente: o grau de exposição dessas cidades ao fenômeno de chuva dirigida, o índice de chuva dirigida crítico para a cidade e a orientação critica das preciptações associadas ao vento. Estes índices podem orientar os critérios de projeto de edificações nestas cidades. Segundo os resultados, para as cidades de Curitiba e Londrina as orientações críticas de fachada são Leste e Nordeste e para Maringá, Norte e Nordeste.
\end{abstract}

Palavras-chave: Chuva Dirigida; Nível de Exposição; Índice de Chuva Dirigida Direcional; Durabilidade de Fachadas.

Abstract: Establishing methods to analyse the influence of environmental conditions on structures is essential when considering the progressive concern with structures' durability. In this context, the present work aims to study the phenomenon of wind-driven rain (rain associated with wind) and its influence on durability of facades. The analysis was based on data from meteorological stations of three different cities in Paraná state: Londrina, Maringá and Curitiba. It sought to identify the most critical directions of wind-driven rain in those cities. The rainfall variation over time was analysed using annual, monthly and directional driving rain indexes, which indicate, respectively: those cities' degree of exposure to wind-driven rain phenomenon, the city's critical driving rain index and the critical orientation of precipitations in association with wind. Those indexes may guide building design criteria for those cities. According to results, for the cities of Curitiba and Londrina the critical facade orientations are East and Northeast while for Maringá, they are North and Northeast.

Keywords: Wind-Driven Rain; Exposure Level; Directional Driving Rain Index; Facade Durability. 
BATISTA, G. D. M.; ET AL., ANÁLISE DO ÍNDICE DE CHUVA DIRIGIDA EM CIDADES DO PARANÁ E SUA IMPORTÂNCIA NO PROJETO DE FACHADAS DE EDIFÍCIOS. $2^{\circ}$ Simpósio Paranaense de Patologia das Construções (20 SPPC), artigo 2SPPC1017, pp. 199-212, 2017. DOI: 10.4322/2SPPC.2017.017

\section{Introdução}

Nas últimas décadas, a durabilidade das construções tem sido foco crescente de pesquisas na Engenharia Civil. Motivados pela busca de edificações que mantenham suas condições de segurança e usabilidade por mais tempo, engenheiros civis tem buscado entender os processos de degradação das edificações e como atuar para mitigá-los.

A durabilidade de uma edificação está diretamente relacionada com os agentes ambientais que causam a deterioração dos seus componentes, sendo a chuva uma das principais causas de manifestações patológicas em ambientes externos. Dentro deste contexto está inserido o estudo das chuvas dirigidas, ou seja, quando há presença associada de precipitação e vento. Esse fenômeno possui alta variabilidade, pois é função da matriz de ventos e chuvas de cada região, o que depende diretamente do clima e topografia do local. Por essa característica, a chuva dirigida faz com que edificações com fachadas idênticas, mas orientações distintas, apresentem durabilidade variável às manifestações patológicas causadas por umedecimento.

Portanto este trabalho se propõe a analisar os dados referentes às chuvas e ventos de três grandes cidades do estado do Paraná: Londrina, Maringá e Curitiba. O estudo visa apresentar as orientações mais críticas para o fenômeno de chuva associada a vento e os graus de agressividade para as diferentes cidades. Assim, engenheiros civis, arquitetos e profissionais da construção terão em mãos uma variável importante que pode balizar as escolhas de projeto, tais como decisões relativas aos materiais, soluções arquitetônicas e orientação das fachadas, em prol da maior durabilidade das edificações.

\section{Referencial Teórico}

Segundo Blocken e Carmeliet [1], chuva dirigida é a chuva (precipitação pluviométrica) que recebe uma componente de velocidade horizontal pela ação do vento e então passa a cair obliquamente. É a chuva carregada pelo vento e, em uma definição mais restrita, é lançada de encontro ao envelope da edificação [2]. Pode ainda ser definida como a quantidade de chuva com uma componente horizontal que passa através de uma superfície vertical pela ação do vento [3].

\subsection{Chuva dirigida e durabilidade de fachadas}

A fachada - como elemento fundamental da envoltória do edifício - age como barreira protetora aos agentes atmosféricos e, assim sendo, sofre com uma das agressões físicas mais importantes: a da água da chuva [4]. Para edificações, a chuva dirigida é uma grande fonte de umidade, afetando seu desempenho higrotérmico e a durabilidade de suas fachadas [3].

De acordo com Choi [5], danos causados pela penetração de água nas fachadas de edifícios tem há tempos sido reconhecidos como um grande problema de manutenção. Estes danos e seus decorrentes prejuízos financeiros podem ser atribuídos a dois tipos de processos. O primeiro está relacionado à penetração de água da chuva para 0 ambiente interior através das fachadas, causando 
BATISTA, G. D. M.; ET AL., ANÁLISE DO ÍNDICE DE CHUVA DIRIGIDA EM CIDADES DO PARANÁ E SUA IMPORTÂNCIA NO PROJETO DE FACHADAS DE EDIFÍCIOS. $2^{\circ}$ Simpósio Paranaense de Patologia das Construções (20 SPPC), artigo 2SPPC1017, pp. 199-212, 2017. DOI: 10.4322/2SPPC.2017.017

inconvenientes aos habitantes, danificando acabamentos do edifício e bens em seu interior. O segundo está relacionado à entrada de umidade nos materiais que constituem a envoltória do edifício, danificando-os. O primeiro processo usualmente ocorre em curta duração de tempo e a degradação depende da intensidade da chuva dirigida. Já o segundo mecanismo é um processo de longo prazo e a degradação está relacionada a quantidade total de chuva dirigida ao longo desse período. Ainda como consequência da chuva dirigida, o aumento da umidade nas paredes externas aumenta a condutividade térmica destas, tornando-as mais permeáveis ao calor [6].

$\mathrm{Na}$ ausência de vento, as gotas de chuva cairiam verticalmente e causariam pouco umedecimento dos envoltórios dos edifícios. Contudo, sob a ação da velocidade do vento, o impacto da chuva dirigida em paredes exteriores passa a submeter as fachadas a diferentes níveis de umidade, conforme ilustrado na Figura 1 [7].

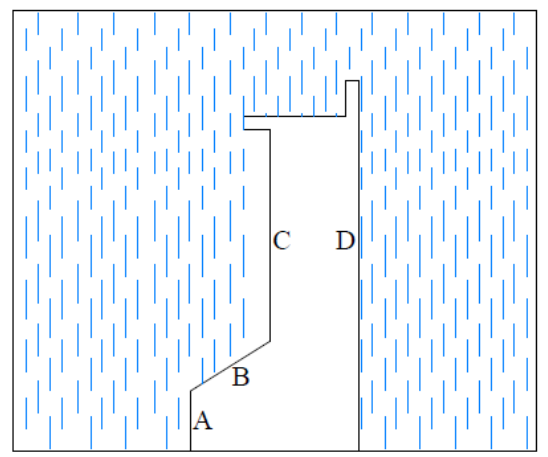

(a)

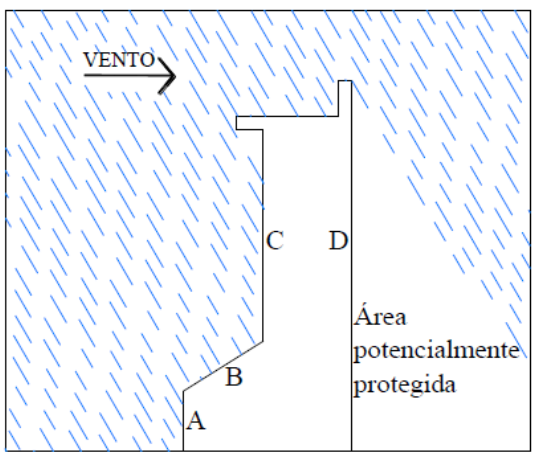

(b)

Figura 1: Influência do vento na direção da chuva dirigida e na exposição das fachadas: (a) Condição sem vento e (b) Condição com vento [7]

De acordo com Giongo, Padaratz e Lamberts [3] e Melo Jr e Carasek [8], os danos causados por esta umidade em abundância vão desde problemas estéticos até degradações profundas. Entre eles estão a proliferação de micro-organismos, a descoloração dos revestimentos, a formação de manchas e eflorescências, a variação dimensional de elementos construtivos, a corrosão eletroquímica dos metais e infiltrações, levando à degradação dos materiais que constituem os componentes das fachadas.

\subsection{Indice de chuva dirigida (ICD anual, ICD direcional e ICD mensal)}

A medição direta da chuva dirigida (medição da quantidade de água passando por um plano vertical) claramente seria a maneira mais natural de quantificar cargas de chuva dirigida em diferentes localidades. Contudo, o equipamento necessário para isso (medidor de parede vertical ou "driving rain gauge") não é um equipamento padrão em estações meteorológicas e, portanto, a chuva dirigida não é medida de maneira rotineira [9]. Seria, então, interessante que a chuva dirigida fosse mensurada através de dados usualmente lidos nas estações. Tendo isso em vista, pesquisadores verificaram a relação proporcional entre a quantidade de chuva dirigida e as variáveis climáticas de influência como velocidade do vento, direção do vento e precipitação estas sendo medições realizadas em estações padrão -, o que conduziu aos métodos semi-empíricos de medição [1]. Estes métodos baseiam-se em relações entre dados 
BATISTA, G. D. M.; ET AL., ANÁLISE DO ÍNDICE DE CHUVA DIRIGIDA EM CIDADES DO PARANÁ E SUA IMPORTÂNCIA NO PROJETO DE FACHADAS DE EDIFÍCIOS. $2^{\circ}$ Simpósio Paranaense de Patologia das Construções (20 SPPC), artigo 2SPPC1017, pp. 199-212, 2017. DOI: 10.4322/2SPPC.2017.017

de vento e precipitações pluviométricas obtidos de estações meteorológicas para o cálculo da chuva dirigida e suas direções principais [10].

Segundo Blocken e Carmeliet [1], nos anos 60, o CIB (Conseil International du Bâtiment) estabeleceu o índice de chuva dirigida (ICD) como sendo o produto entre a velocidade média do vento e a quantidade total de precipitação, conforme a Equação 1.

$$
I C D=V \times \frac{P}{1000}
$$

Onde:

ICD = índice de chuva dirigida $\left(\mathrm{m}^{2} / \mathrm{s}\right)$

$\mathrm{V}=$ velocidade média do vento $(\mathrm{m} / \mathrm{s})$

$\mathrm{P}=$ precipitação total $(\mathrm{mm})$

Quando o ICD é calculado a partir de médias anuais de velocidade do vento e de precipitação acumulada anual - ou seja, baseando-se em dados de anos completos ele é denominado ICD anual (ou ICDa), sendo este um método razoavelmente preciso para comparação entre o total acumulado de chuva dirigida em paredes de diferentes lugares [3]. Dessa forma, este parâmetro é importante como um critério global de balizamento de projetos de fachadas de edifícios de modo a torná-las mais duráveis, embora não seja comumente considerado na prática de projeto [10].

Sabe-se que a incidência de vento tem sentidos preferenciais para cada localidade e isso resulta em níveis de exposição à chuva dirigida diferentes para as várias orientações de uma fachada. Sendo assim, o ICDa generaliza em tal grau a informação que não orienta o projetista na especificação de soluções diferentes para as distintas orientações das fachadas de um edifício, não lhe permitindo racionalizar o projeto da fachada da melhor maneira possível. Tendo isso em vista, idealizou-se o ICD direcional (ou ICDd), que separa a intensidade de chuva dirigida incidente em cada sentido, permitindo quantificar o nível de agressividade pela chuva dirigida para cada orientação de uma fachada [10].

Empregando a mesma Equação 1 calcula-se o ICD para cada orientação do vento (Norte, Nordeste, Leste, Sudeste, Sul, Sudoeste, Oeste e Noroeste). Para realizar o cálculo, utiliza-se a precipitação total dos dias em que o vento foi predominante na orientação em questão e a velocidade média anual do vento nesta mesma direção, atentando ao fato de que em dias de precipitação nula a velocidade do vento não entra no cálculo de sua média.

Esse índice direcional facilita a visualização e a análise da influência de cada direção quanto à exposição à chuva dirigida, uma vez que podem ser feitos gráficos do tipo roseta, como mostrado na Figura 2, o que facilita a visualização do grau de exposição para cada direção de fachada [11]. 


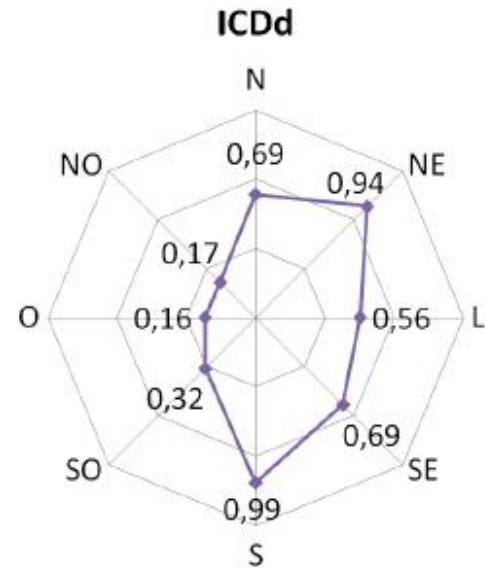

Figura 2: Gráfico tipo roseta do ICDd [11]

A importância do ICDd encontra-se no fato de que este pode ser utilizado para fundamentar o uso de materiais mais resistentes a umidade em fachadas mais críticas de um mesmo edifício, de modo a otimizar o desempenho dos materiais empregados [11]. Dessa maneira, materiais mais nobres e uma execução mais cuidadosa devem ser utilizados nas fachadas onde o ICDd é mais elevado - no exemplo da Figura 2, estas seriam as fachadas voltadas às direções Sul e Nordeste, respectivamente. Já nas fachadas em que o ICDd é mais baixo, os materiais usados para o revestimento da fachada podem ser menos nobres, o que pode implicar em redução dos custos, sem comprometer a durabilidade e eficiência da edificação [10].

Ainda é possível calcular índices de chuva dirigida mensais (ICD mensais) para se fazer uma comparação do comportamento da chuva dirigida durante o ano [3]. Com isso pode-se verificar qual é a chuva dirigida crítica que um local recebe, ou seja, qual é a carga máxima de chuva dirigida que uma edificação será submetida ao longo dos meses de um ano. Esse valor pode ainda ser utilizado como a carga máxima de projeto da edificação [10].

\subsection{Classes de exposição}

Com a finalidade de gerar parâmetros de referência para classificar o nível de impacto da chuva dirigida em uma localidade quanto ao Índice de Chuva Dirigida (ICD), foram criadas por pesquisadores faixas distintas de exposição. Com isso, a partir do ICD anual, pode-se classificar uma localidade quanto ao seu grau de exposição.

A primeira classificação foi proposta por Lacy [12], englobando três faixas (Protegido, Moderado e Severo), de acordo com ICDa da localidade.

Em 2002, Chand e Bhargava [6] propuseram uma nova faixa de classificação no critério de Lacy [12], devido aos altos índices de chuva dirigida encontrados na Índia.

A Tabela 1 mostra a classificação proposta. 
BATISTA, G. D. M.; ET AL., ANÁLISE DO ÍNDICE DE CHUVA DIRIGIDA EM CIDADES DO PARANÁ E SUA IMPORTÂNCIA NO PROJETO DE FACHADAS DE EDIFÍCIOS. $2^{\circ}$ Simpósio Paranaense de Patologia das Construções (20 SPPC), artigo 2SPPC1017, pp. 199-212, 2017. DOI: 10.4322/2SPPC.2017.017

Tabela 1: Classificação segundo o grau de exposição por Chand e Bhargava [6]

\begin{tabular}{cc}
\hline $\begin{array}{c}\text { Índice de Chuva Dirigida } \\
\left(\mathbf{m}^{2} / \mathbf{s}\right)\end{array}$ & Grau de Exposição \\
\hline$I C D \leq 3$ & Protegido \\
$3 \leq I C D \leq 7$ & Moderado \\
$7 \leq I C D \leq 11$ & Alto \\
$I C D>11$ & Severo \\
\hline
\end{tabular}

Por se tratar de uma classificação mais recente, utilizou-se no presente trabalho o critério de Chand e Bhargava [6].

\section{Metodologia}

Para este trabalho, foi utilizado o método de cálculo semiempírico, que consiste na análise dos dados de precipitação e velocidade do vento. Os objetos de estudo foram 3 cidades do Paraná, cujos dados meteorológicos foram obtidos através do SIMEPAR (Sistema Meteorológico do Paraná), para uma série histórica de Janeiro de 2006 até Dezembro de 2015.

Os dados obtidos consistem de precipitação, em milímetros de chuva, velocidade do vento média, em $\mathrm{m} / \mathrm{s}$, e direção do vento, variando a cada $45^{\circ}$ para cada sentido da rosa dos ventos, sendo o Norte equivalente a $0^{\circ}$, e crescendo no sentido horário. É importante ressaltar que os arquivos de precipitação obtidos abrangem todos os dias do ano, o que inclui aqueles em que a precipitação foi igual a zero. Sendo assim, foi necessário desconsiderar estes dias com valores nulos, visto que para o cálculo do Índice de Chuva Dirigida, é necessário que haja precipitação.

Para o cálculo do ICDa, determinou-se a precipitação total para cada ano, assim como as respectivas velocidades médias, e aplicou-se a Equação 1. O nível de exposição de cada cidade, de acordo com o critério de Chand e Bhargava [6], foi determinado através da média de todos os anos analisados durante este período.

O cálculo também foi realizado para uma divisão de dados mensal, de modo que foi possível determinar o mês mais crítico ao longo dos anos. Para este caso, foram calculadas a soma da precipitação mensal e a velocidade média mensal, de modo a obter as variáveis para a Equação 1 e estabelecer o ICD mensal. Em seguida, calculou-se a média da série de anos analisada, para estipular o nível de exposição do mês correspondente.

Para o Índice de Chuva Dirigida Direcional, foram necessárias algumas adaptações nos dados. Uma vez que o arquivo de direção do vento indicava os pontos cardeais e colaterais em graus, foi preciso alterar os valores para a direção correspondente, como mostra a Figura 3. 
BATISTA, G. D. M.; ET AL., ANÁLISE DO ÍNDICE DE CHUVA DIRIGIDA EM CIDADES DO PARANÁ E SUA IMPORTÂNCIA NO PROJETO DE FACHADAS DE EDIFÍCIOS. $2^{\circ}$ Simpósio Paranaense de Patologia das Construções (20 SPPC), artigo 2SPPC1017, pp. 199-212, 2017. DOI: 10.4322/2SPPC.2017.017

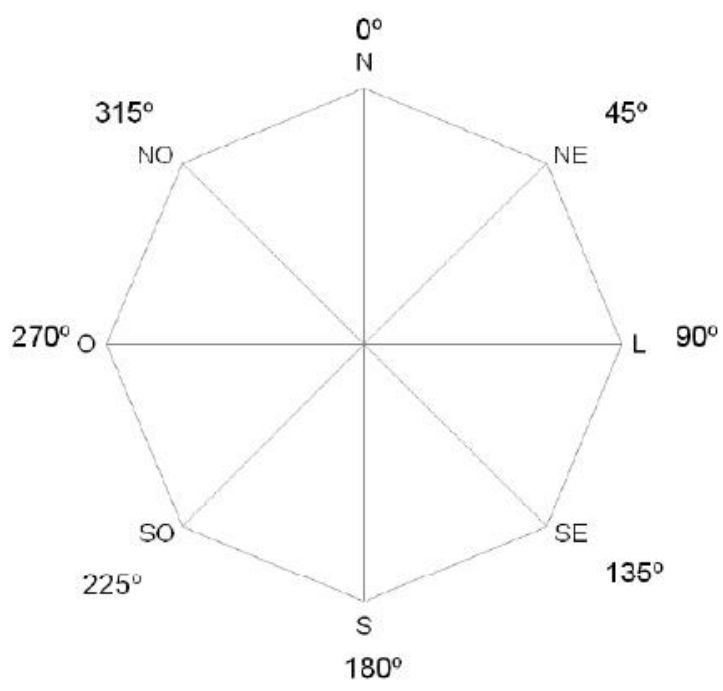

Figura 3: Direção dos ventos expressas em graus, para o cálculo do Índice de

Chuva Dirigida Direcional

Foram então separados, para cada direção, a precipitação total e a velocidade média do vento, e realizado o cálculo do ICDd através da Equação 1 para cada ano. Por fim, fez-se a média da série histórica e determinou-se a direção com o maior nível de exposição para cada cidade analisada.

Além das tabelas com os cálculos, os resultados também foram expressos na forma de gráficos, para melhor entendimento e análise.

\section{Resultados}

\subsection{ICDa}

As Tabela 2, 3 e 4 mostram os valores obtidos de precipitação anual, velocidade média do vento e ICDa, para as cidades de Curitiba, Londrina e Maringá, para os anos de 2006 a 2015, além do nível de exposição, segundo os critérios de Chand e Bhargava [6].

Tabela 2: Precipitação anual, velocidade média do vento e ICDa para Curitiba

\begin{tabular}{|c|c|c|c|c|}
\hline \multicolumn{5}{|c|}{ Curitiba } \\
\hline Ano & $\begin{array}{c}\text { Veloc. Média } \\
(\mathrm{m} / \mathrm{s})\end{array}$ & $\begin{array}{l}\text { Precipitação Anual } \\
\text { (mm) }\end{array}$ & $\begin{array}{l}\text { ICDa } \\
\left(\mathrm{m}^{2} / \mathrm{s}\right)\end{array}$ & $\begin{array}{c}\text { Nível de Exposição - } \\
\text { Chand \& Bhargava (2002) }\end{array}$ \\
\hline 2006 & 2,052 & 916,400 & 1,88 & Protegido \\
\hline 2007 & 2,188 & 1241,800 & 2,72 & Protegido \\
\hline 2008 & 2,046 & 1182,800 & 2,42 & Protegido \\
\hline 2009 & 2,034 & 1645,400 & 3,35 & Moderado \\
\hline 2010 & 2,031 & 1761,800 & 3,58 & Moderado \\
\hline 2011 & 2,052 & 1841,400 & 3,78 & Moderado \\
\hline 2012 & 2,047 & 1467,400 & 3,00 & Moderado \\
\hline 2013 & 2,111 & 1411,600 & 2,98 & Protegido \\
\hline 2014 & 2,077 & 1573,200 & 3,27 & Moderado \\
\hline 2015 & 2,003 & 1835,200 & 3,68 & Moderado \\
\hline \multicolumn{3}{|c|}{ ICD médio (2006 - 2015) } & 3,065 & Moderado \\
\hline
\end{tabular}


BATISTA, G. D. M.; ET AL., ANÁLISE DO ÍNDICE DE CHUVA DIRIGIDA EM CIDADES DO PARANÁ E SUA IMPORTÂNCIA NO PROJETO DE FACHADAS DE EDIFÍCIOS. $2^{\circ}$ Simpósio Paranaense de Patologia das Construções (20 SPPC), artigo 2SPPC1017, pp. 199-212, 2017. DOI: 10.4322/2SPPC.2017.017

Tabela 3: Precipitação anual, velocidade média do vento e ICDa para Londrina

\begin{tabular}{|c|c|c|c|c|}
\hline \multicolumn{5}{|c|}{ Londrina } \\
\hline Ano & $\begin{array}{c}\text { Veloc. Média } \\
(\mathrm{m} / \mathrm{s})\end{array}$ & $\begin{array}{c}\text { Precipitação Anual } \\
(\mathrm{mm})\end{array}$ & $\begin{array}{l}\text { ICDa } \\
\left(\mathrm{m}^{2} / \mathrm{s}\right)\end{array}$ & $\begin{array}{c}\text { Nível de Exposição - } \\
\text { Chand \& Bhargava (2002) }\end{array}$ \\
\hline 2006 & 2,313 & 1054,800 & 2,44 & Protegido \\
\hline 2007 & 2,329 & 1305,800 & 3,04 & Moderado \\
\hline 2008 & 2,373 & 1288,200 & 3,06 & Moderado \\
\hline 2009 & 2,200 & 2442,200 & 5,37 & Moderado \\
\hline 2010 & 2,325 & 1768,000 & 4,11 & Moderado \\
\hline 2011 & 2,334 & 1440,400 & 3,36 & Moderado \\
\hline 2012 & 2,298 & 1433,800 & 3,30 & Moderado \\
\hline 2013 & 2,381 & 1878,800 & 4,47 & Moderado \\
\hline 2014 & 2,391 & 1378,200 & 3,30 & Moderado \\
\hline 2015 & 2,200 & 2441,600 & 5,37 & Moderado \\
\hline \multicolumn{3}{|c|}{ ICD médio (2006 - 2015) } & 3,782 & Moderado \\
\hline
\end{tabular}

Tabela 4: Precipitação anual, velocidade média do vento e ICDa para Maringá

\begin{tabular}{|c|c|c|c|c|}
\hline \multicolumn{5}{|c|}{ Maringá } \\
\hline Ano & $\begin{array}{l}\text { Veloc. Média } \\
(\mathrm{m} / \mathrm{s})\end{array}$ & \begin{tabular}{|c|}
$\begin{array}{c}\text { Precipitação Anual } \\
(\mathrm{mm})\end{array}$ \\
\end{tabular} & $\begin{array}{l}\mathrm{ICDa} \\
\left(\mathrm{m}^{2} / \mathrm{s}\right)\end{array}$ & $\begin{array}{c}\text { Nível de Exposição - } \\
\text { Chand \& Bhargava (2002) }\end{array}$ \\
\hline 2006 & 2,639 & 1220,800 & 3,22 & Moderado \\
\hline 2007 & 2,724 & 1289,800 & 3,51 & Moderado \\
\hline 2008 & 2,559 & 1182,000 & 3,03 & Moderado \\
\hline 2009 & 2,316 & 1739,000 & 4,03 & Moderado \\
\hline 2010 & 2,499 & 1214,200 & 3,03 & Moderado \\
\hline 2011 & 2,671 & 1637,600 & 4,37 & Moderado \\
\hline 2012 & 2,616 & 1742,600 & 4,56 & Moderado \\
\hline 2013 & 2,678 & 1747,200 & 4,68 & Moderado \\
\hline 2014 & 2,718 & 1840,400 & 5,00 & Moderado \\
\hline 2015 & 2,608 & 2527,200 & 6,59 & Moderado \\
\hline & ICD médio & D15) & 4,203 & Moderado \\
\hline
\end{tabular}

Ao analisar as três cidades, percebe-se claramente um aumento do valor do ICDa ao longo dos anos. Em Curitiba, o valor máximo foi de 3,78 $\mathrm{m}^{2} / \mathrm{s} \mathrm{em} \mathrm{2011,} \mathrm{enquanto} \mathrm{em}$ Londrina foi de $5,37 \mathrm{~m}^{2} / \mathrm{s} \mathrm{em} 2009$. Já para Maringá, o máximo atingido foi $6,59 \mathrm{~m}^{2} / \mathrm{s}$, em 2015. Para os três municípios, o nível de exposição médio para este período, segundo o critério de Chand e Bhargava [6] é Moderado, com valores de 3,065 m²/s, $3,782 \mathrm{~m}^{2} / \mathrm{s}$ e $4,203 \mathrm{~m}^{2} / \mathrm{s}$, respectivamente.

$\mathrm{Na}$ Figura 4 observa-se esta evolução do ICDa ao longo dos anos para as três cidades. Seria necessário um período de dados mais longo para entender melhor o comportamento climático das cidades e, consequentemente, fazer uma análise mais completa. 


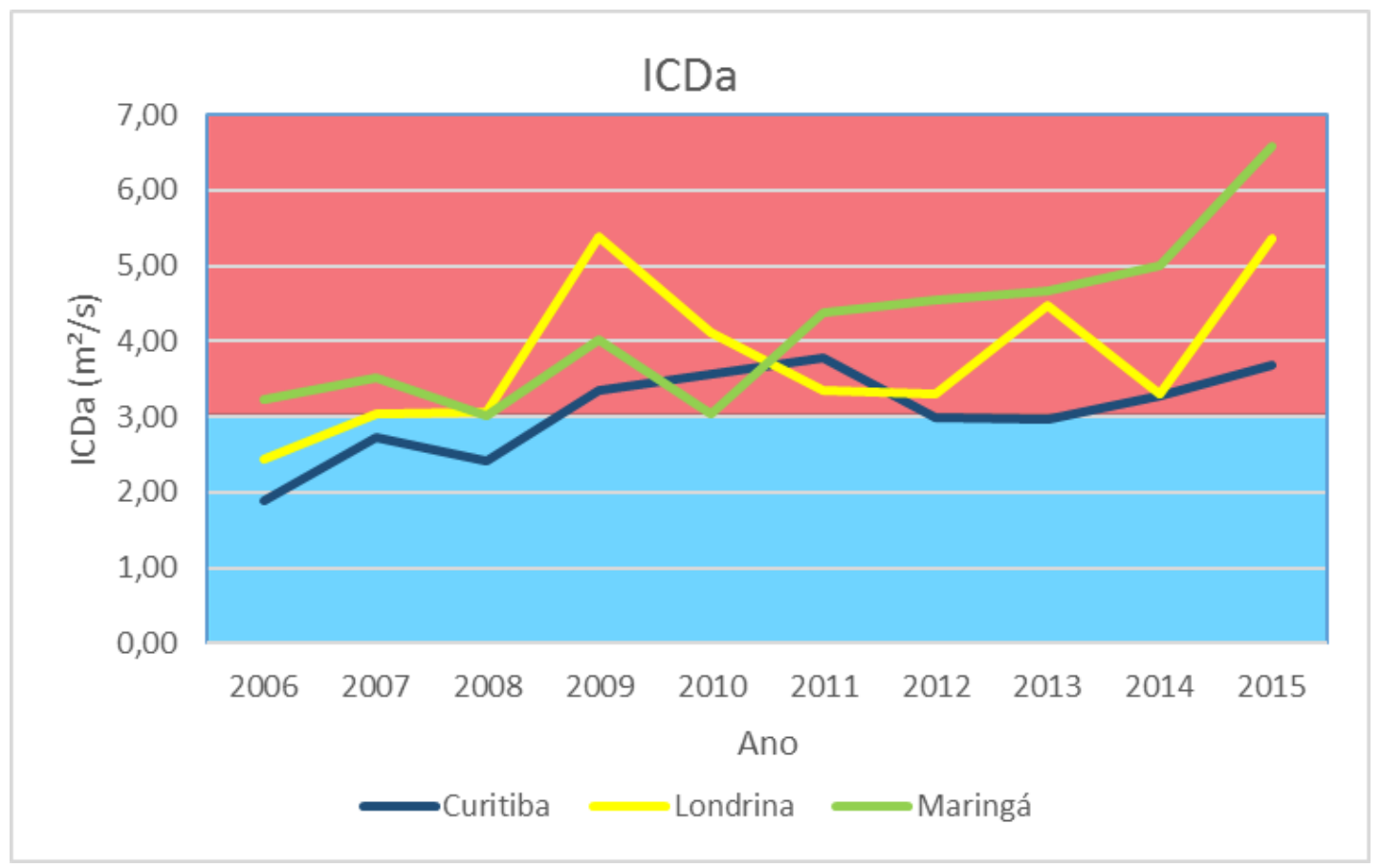

Figura 4: Variação do ICDa em Curitiba, Londrina e Maringá

Para o período de 2006 a 2015, percebe-se que na cidade de Londrina ocorrem três picos no valor do ICDa, nos anos de 2009, 2013 e 2015, que diferem do comportamento característico da cidade e ficam consideravelmente acima da média. Já para a cidade de Maringá, observa-se um crescimento regular ao longo dos anos, com leves quedas em 2008 e 2010, até atingir seu valor máximo em 2015. No caso da capital paranaense, o ano de 2006 foi o que teve o menor ICDa, com o valor de $1,88 \mathrm{~m}^{2} / \mathrm{s}$, localizando-se na faixa de exposição protegida. A partir de 2009, o índice passou para Moderado com um pico em 2011, seguido por uma queda, mas com novo crescimento até o ano de 2015.

\subsection{ICD mensal}

A Figura 5 apresenta os resultados obtidos para a média de ICD mensais dos 12 meses para as cidades de Curitiba, Londrina e Maringá. Para as três cidades os maiores índices (ICDs críticos) ocorrem para o mês de Janeiro - 0,39, 0,48 e 0,46 $\mathrm{m}^{2} / \mathrm{s}$, respectivamente. Seguindo Janeiro, os meses de Novembro e Dezembro são aqueles que apresentam maior ICD mensais para as três localidades. 
BATISTA, G. D. M.; ET AL., ANÁLISE DO ÍNDICE DE CHUVA DIRIGIDA EM CIDADES DO PARANÁ E SUA IMPORTÂNCIA NO PROJETO DE FACHADAS DE EDIFÍCIOS. $2^{\circ}$ Simpósio Paranaense de Patologia das Construções (20 SPPC), artigo 2SPPC1017, pp. 199-212, 2017. DOI: 10.4322/2SPPC.2017.017

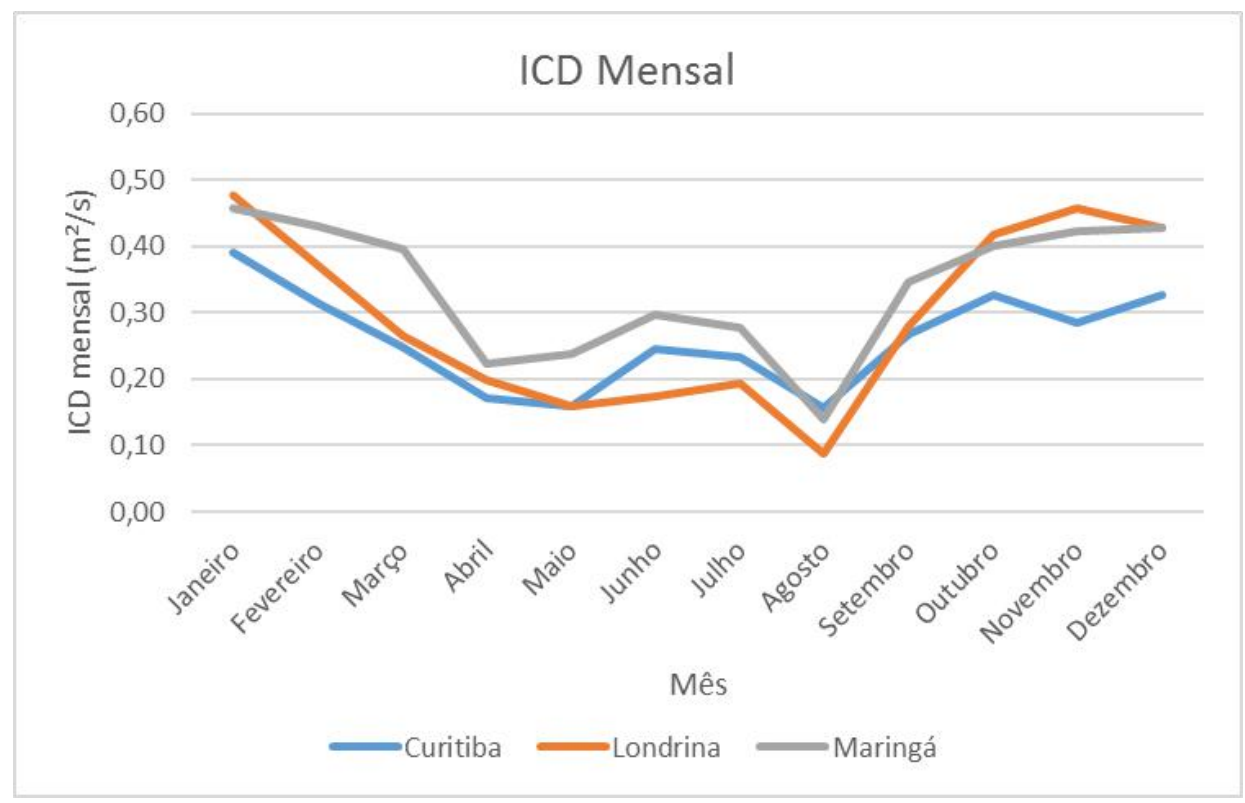

Figura 5: Média de ICD mensal da série histórica analisada para Curitiba, Londrina e Maringá

\subsection{ICDd}

As Figura 6, 7 e 8 mostram a média da incidência da chuva dirigida direcional para cada uma das três cidades estudadas, no período de 2006 a 2015.

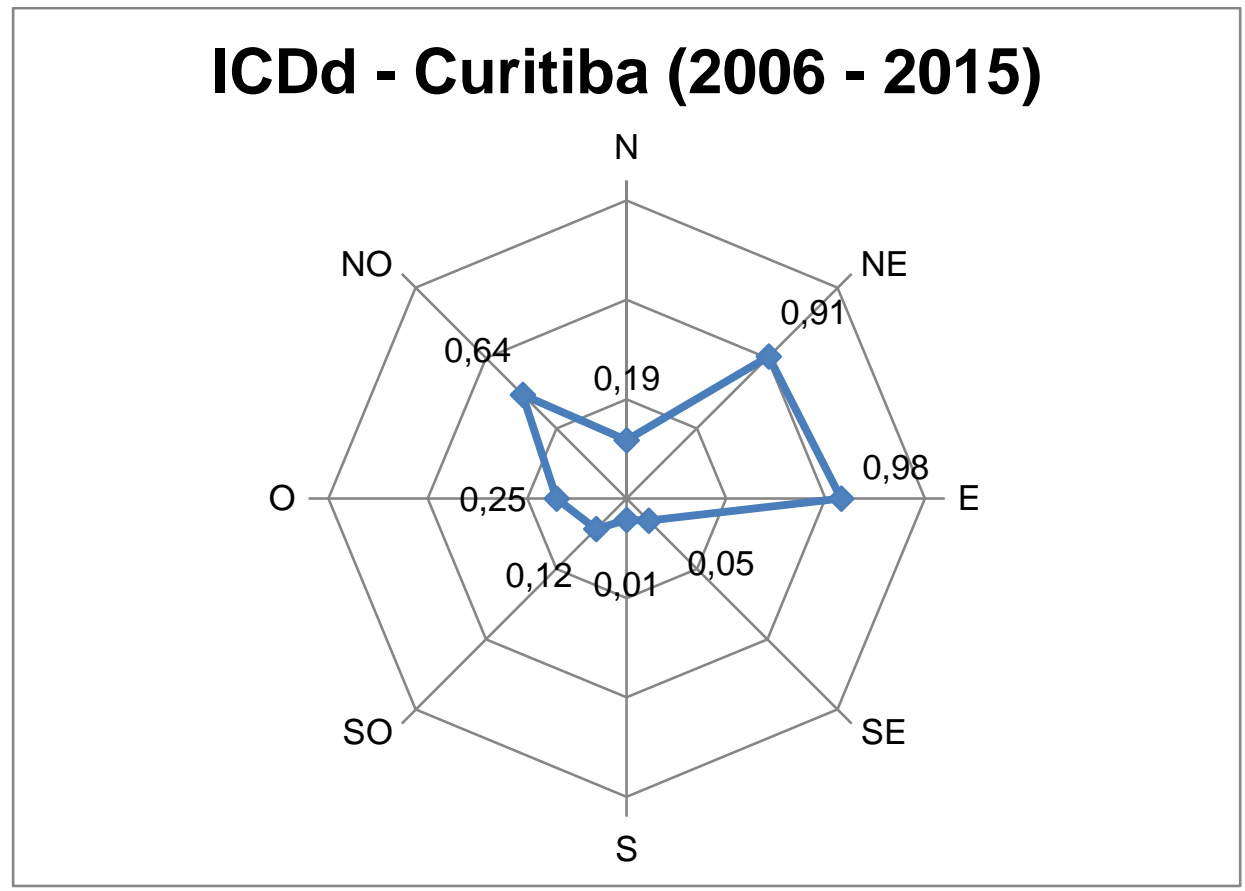

Figura 6: ICDd médio de Curitiba entre 2006 e 2015 


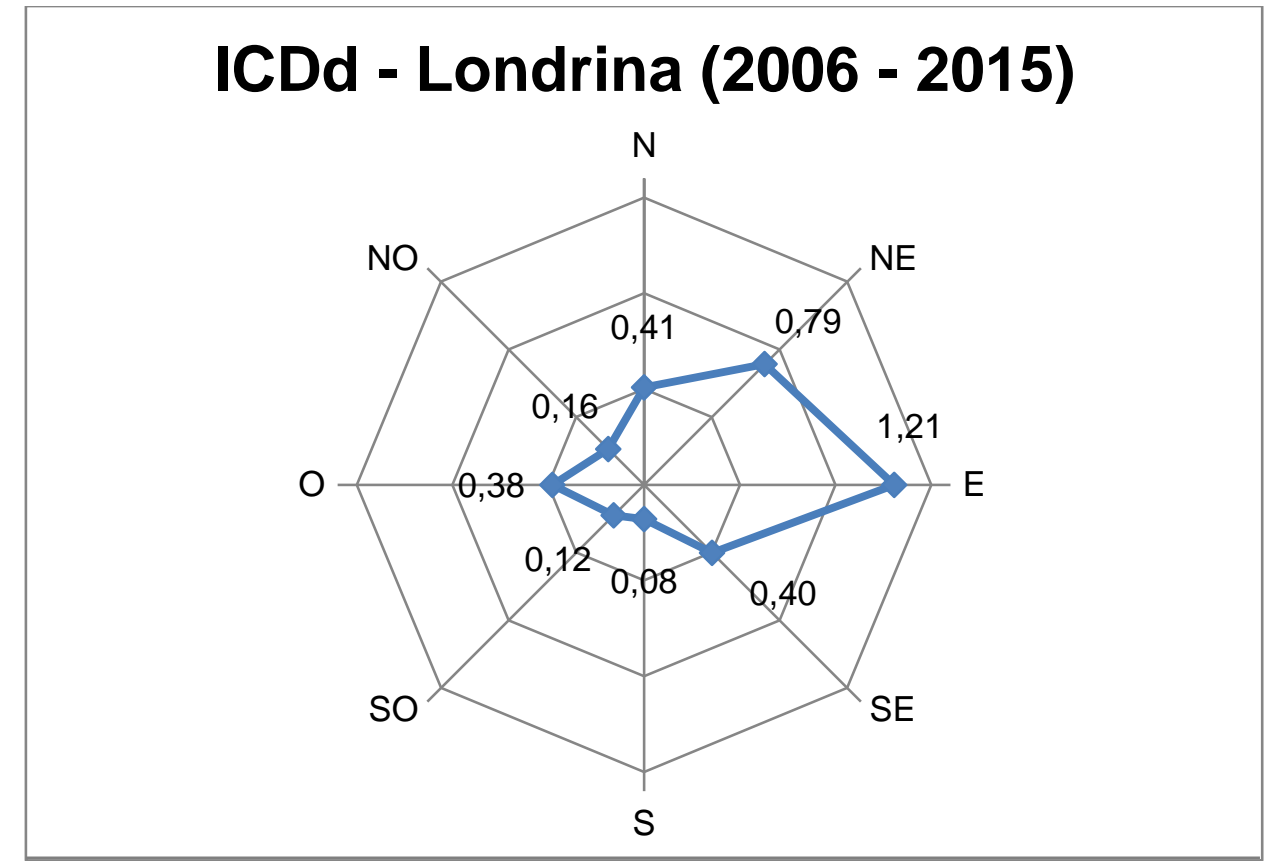

Figura 7: ICDd médio de Londrina entre 2006 e 2015

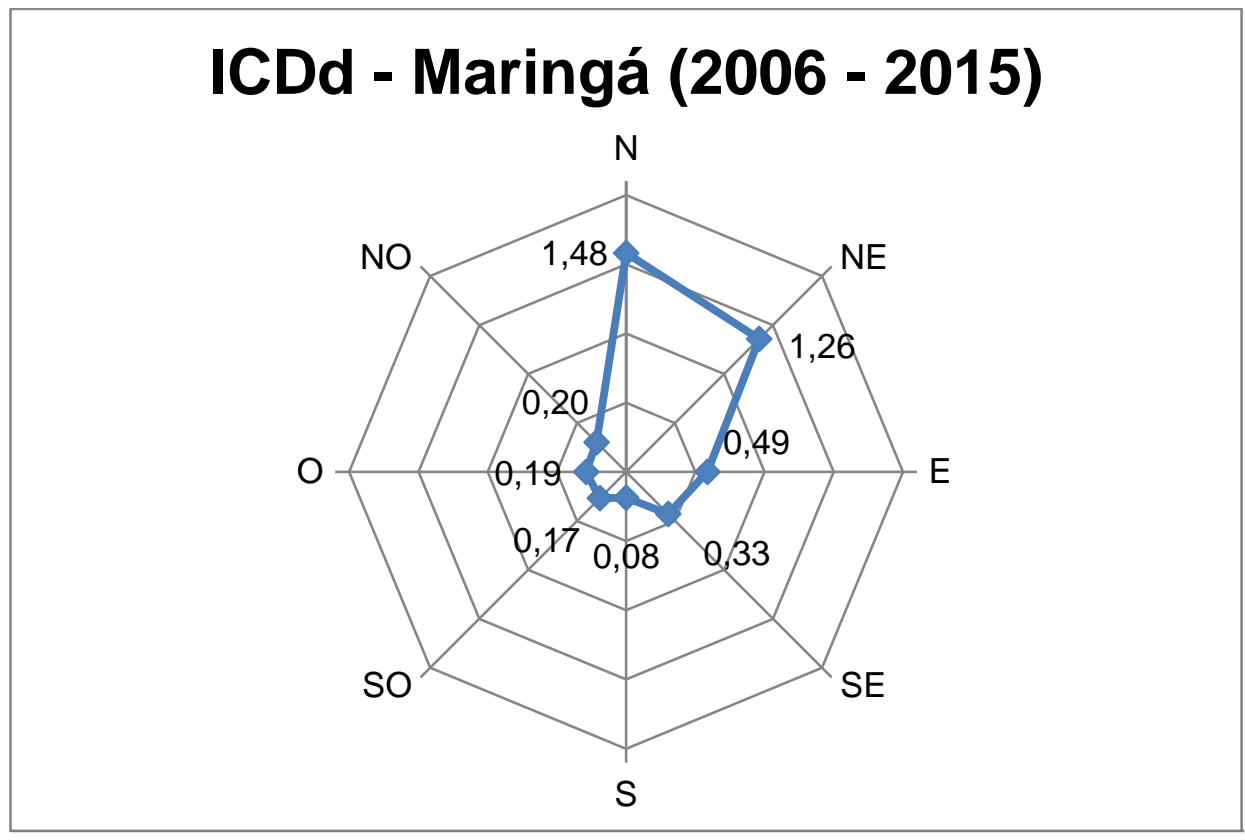

Figura 8: ICDd médio de Maringá entre 2006 e 2015

Para a cidade de Curitiba, a direção com o maior ICDd foi a Leste, com um valor de $0,98 \mathrm{~m}^{2} / \mathrm{s}$. Já a direção menos crítica foi a Sul, com valor quase nulo de $0,01 \mathrm{~m}^{2} / \mathrm{s}$. Também é importante notar que a direção Nordeste também apresentou um valor relativamente alto para a cidade, com $0,91 \mathrm{~m}^{2} / \mathrm{s}$.

Para Londrina, a direção mais crítica também foi a Leste, com um valor de 1,21 $\mathrm{m}^{2} / \mathrm{s}$, seguida, assim como em Curitiba, pela direção Nordeste, com $0,79 \mathrm{~m}^{2} / \mathrm{s}$. $\mathrm{O}$ valor menos crítico foi $0,08 \mathrm{~m}^{2} / \mathrm{s}$, na direção Sul. 
BATISTA, G. D. M.; ET AL., ANÁLISE DO ÍNDICE DE CHUVA DIRIGIDA EM CIDADES DO PARANÁ E SUA IMPORTÂNCIA NO PROJETO DE FACHADAS DE EDIFÍCIOS. $2^{\circ}$ Simpósio Paranaense de Patologia das Construções (20 SPPC), artigo 2SPPC1017, pp. 199-212, 2017. DOI: 10.4322/2SPPC.2017.017

Já para Maringá, a direção Norte se mostrou a mais problemática, com um ICDd de $1,48 \mathrm{~m}^{2} / \mathrm{s}$, seguida pela direção Nordeste, com 1,26 m²/s. O menor valor foi para a direção Sul, com $0,08 \mathrm{~m}^{2} / \mathrm{s}$.

\section{Considerações Finais}

Com os índices de chuva dirigida anuais médios obtidos pelos dados do SIMEPAR, as três maiores cidades do estado do Paraná puderam ser consideradas de exposição moderada quanto ao fenômeno de chuva dirigida, segundo o critério de Chand e Bhargava [6]. Tendo em conta que, sob o ponto de vista de escolhas de projeto, analisar apenas o ICDa de uma região leva a assunção de que o nível de agressividade é uniforme para todas as direções de fachada, os índices de chuva dirigida direcionais médios para cada uma das três cidades foram obtidos, gerando gráficos do tipo roseta. Esta ferramenta de fácil visualização pode permitir que projetitas destas três cidades consigam distinguir quais direções de fachada serão as mais críticas em suas edificações futuras, permitindo-Ihes discretizar quais fachadas necessitam de materiais mais resistentes a umidade e deverão ter execução mais cautelosa, de maneira que recursos financeiros sejam empenhados somente na medida do necessário. Isso permitirá a racionalização do projeto e a consequente redução de custos. Para Curitiba, as direções de fachada que demandariam mais atenção são Leste e Nordeste, respectivamente. O mesmo acontece para Londrina. Já para a cidade de Maringá, as direções mais críticas ao projeto e execução são Norte e Nordeste, nesta ordem.

Pela obtenção do ICD crítico (maior ICD mensal) para cada cidade, pode-se concluir que Janeiro é o mês com o maior índice de chuva dirigida das três localidades - 0,39 $\mathrm{m}^{2} / \mathrm{s}$ para Curitiba, 0,48 m²/s para Londrina e $0,46 \mathrm{~m}^{2} / \mathrm{s}$ para Maringá. No momento de elaboração de projetos de fachadas, além do nível de exposição da cidade como um todo (ICDa), o ICD crítico deve ser levado em consideração, pois corresponde à maior incidência de chuva dirigida que a edificação vai receber durante todo o ano, podendo ainda ser utilizado como a carga máxima de projeto para chuva dirigida da edificação.

Tendo em vista que a chuva dirigida é um fenômeno climático, esta encontra-se sujeita a anos atípicos e variações imprevisíveis - variações estas que foram apresentadas em forma de gráficos ao longo dos anos da série histórica tomada para análise. Em decorrência dessas variações já esperadas, é recomendado que avaliações sejam feitas preferencialmente com séries históricas de 10 a 30 anos, ou no mínimo 5 anos, pois do contrário o nível real de exposição de uma localidade pode ficar subestimado ou superestimado [3]. Em razão disso, pode-se concluir que a série de dados tomada para análise foi consistente para avaliação das localidades - pois é composta de 10 anos. Contudo, recomenda-se que os índices calculados sejam atualizados ano após ano pela alimentação com novos dados, buscando sempre informações mais completas e que se aproximem ao máximo da realidade das cargas de chuva dirigida a que uma edificação futura será imposta ao longo de sua vida útil.

Além do supracitado, recomenda-se para trabalhos futuros o desenvolvimento de um critério para a classificação do nível de agressividade quanto à chuva dirigida para cada direção de fachada, através do ICDd, uma vez que os critérios de Chand e Bhargava [6] e de Lacy [12] só podem ser associados aos ICD anuais, não podendo 
BATISTA, G. D. M.; ET AL., ANÁLISE DO ÍNDICE DE CHUVA DIRIGIDA EM CIDADES DO PARANÁ E SUA IMPORTÂNCIA NO PROJETO DE FACHADAS DE EDIFÍCIOS. $2^{\circ}$ Simpósio Paranaense de Patologia das Construções (20 SPPC), artigo 2SPPC1017, pp. 199-212, 2017. DOI: 10.4322/2SPPC.2017.017

serem discretizados para cada direção cardeal. Soma-se a isso um possível trabalho de associação entre os tipos de revestimentos de fachadas mais comumente utilizados e os respectivos valores de ICDd que estes revestimentos suportariam para uma vida útil estipulada, de maneira a auxiliar melhor os projetitas na escolha de materiais para fachadas.

\section{Referências}

[1] BLOCKEN, B.; CARMELIET, J. A review of wind-driven rain research in building science. Journal of Wind Engineering and Industrial Aerodynamics, v. 92, n. 13, p. 1079-1130, Nov/ 2004.

[2] MOOK, F. J. R. Driving rain on building envelopes. Bouwstenen series of the Faculty of Architecture, Planning and Building of the Eindhoven University of Technology, 2003.

[3] GIONGO, M.; PADARATZ, I. J.; LAMBERTS, R. Determinação da exposição à chuva dirigida em Florianópolis, SC: índices de chuva dirigida e metodos semi-empíricos. Ambiente Construído, Porto Alegre, v. 11, n. 1, p. 7-23, Jan./Mar. 2011.

[4] POYASTRO, P. C. Influência da volumetria e das condições de entorno da edificação no manchamento e infiltração de água em fachadas por ação de chuva dirigida. 180f. Dissertação de Mestrado - Escola de Engenharia, Universidade Federal do Rio Grande do Sul (UFRGS), Porto Alegre, Brasil, 2011.

[5] CHOI, E. C. C. Wind-driven rain on building faces and the driving-rain index. Journal of Wind Engineering and Industrial Aerodynamics, v.79, n.1 e 2 p 105122, Jan/ 1999.

[6] CHAND, I; BHARGAVA, P. K. Estimation of driving rain index for India. Building and Environment, v. 37, n. 5, p. 549-554, Maio/ 2002.

[7] THOMAZ, E. Manual Técnico de Alvenaria: patologia. São Paulo: Projeto, 1990. p. $97-117$.

[8] MELO JUNIOR, C. M.; CARASEK, H. Índices de chuva dirigida direcional e análise do nível de umedecimento em fachadas de edifício multipavimento em Goiânia, GO. Ambiente Construído, Porto Alegre, v 11, n. 3 p. 23-37, Jul./Set. 2011.

[9] RYDOCK, J. P.; LISO, K. R.; FORLAND, E. J.; NORE, K.; THUE J. V. A driving rain exposure index for Norway. Building and Environment, v 40, n. 11 p. 14501458, Nov./ 2005.

[10] TOMAZ, T. P.; BILL, G. H.; GIORDANO, D. E.; PEREIRA, E.; MEDEIROS, M. H. F. Índice de Chuva Dirigida para o Estado do Paraná: uma ferramenta para projeto de fachadas. In: Lúcia Bressiani; Cristiano Poleto. (Org.). Tópicos 
BATISTA, G. D. M.; ET AL., ANÁLISE DO ÍNDICE DE CHUVA DIRIGIDA EM CIDADES DO PARANÁ E SUA IMPORTÂNCIA NO PROJETO DE FACHADAS DE EDIFÍCIOS. $2^{\circ}$ Simpósio Paranaense de Patologia das Construções ( $2^{\circ} \mathrm{SPPC}$ ), artigo 2SPPC1017, pp. 199-212, 2017. DOI: 10.4322/2SPPC.2017.017

Especiais em Construção Civil. 1ed. Toledo: UTFPR, 2014, v. único, p. 107122.

[11] MARTINEZ, M. A. C. A influência do índice de Chuva Dirigida sobre o grau de exposição das fachadas de edifícios da cidade de Curitiba. Trabalho Final de Curso de Graduação. Universidade Federal do Paraná (UFPR). Curitiba, Brasil, 2013.

[12] LACY, R. E. Climate and Building in Britain: building research establishment. London: Her Majesty's Stationery Office, 1977. 185 p. 